\title{
Ticagrelor Use in Stroke Patients: Past, Present, and Future
}

\section{Rahul Chandra \\ Harneel Saini \\ Russell Cerejo \\ Ashis Tayal \\ Konark Malhotra}

Department of Neurology, Allegheny Health Network, Pittsburgh, PA, USA
Correspondence: Konark Malhotra Department of Neurology, Allegheny Health Network, Pittsburgh, PA, USA $\mathrm{Tel}+|-4| 2-359-884 \mid$

Fax + I- 4I2-442-2II5

Email konark.malhotra@yahoo.com
Abstract: Antiplatelet medications are the mainstay for secondary stroke treatment. Aspirin, clopidogrel, and aspirin-dipyridamole are commonly used antiplatelet medications. Other antiplatelet medications such as ticagrelor and prasugrel have been majorly used in cardiovascular or neuro-interventional specialties. Recent studies have paved a way to their use in secondary stroke prevention. In this review, we have briefly discussed the pharmacology of ticagrelor, published literature in cardiology and stroke trials, use of ticagrelor among patients with ischemic strokes, and compared its efficacy, limitations and side-effects with other antiplatelet medications.

Keywords: Ticagrelor, stroke, clopidogrel, platelet inhibition

\section{Introduction}

Ticagrelor is a potent, direct-acting antiplatelet agent that reversibly binds and inhibits platelet adenosine diphosphate P2Y12 receptors. ${ }^{1}$ In comparison to other P2Y12 antagonists, ticagrelor does not require conversion from a prodrug to an active formulation. Ticagrelor and its metabolite are equipotent and achieve peak concentration within 2-3 days of its last dose. Due to its higher affinity for protein, ticagrelor maintains a linear pharmacokinetics. ${ }^{2}$ Additionally, Ticagrelor is dosed twice-daily which allows for a more consistent inhibition of platelets over a 24-hour period. $^{2}$

\section{Literature Review}

Ticagrelor Use in Non-Stroke Trials

The use of ticagrelor in ischemic events was initially studied in patients with coronary artery disease (CAD).

DISPERSE-2 (2009)

The Dose confIrmation Study assessing anti-platelets Effects of AZD6140 vs clopidogRel in non-ST-segment Elevation myocardial infarction (NSTEMI), DISPERSE, was an international, randomized, double-blinded, prospective trial that evaluated the efficacy of ticagrelor and clopidogrel in patients with NSTEMI. ${ }^{4}$ In comparison to clopidogrel, ticagrelor showed a significant reduction in the rates of vascular deaths without an increase in the overall rate of major bleeding in patients with acute coronary syndrome regardless of ST-segment elevation. The primary endpoint looked at the rate of major and/or minor bleeding complications. The primary endpoint was $8.1 \%$ in the clopidogrel group and 
$9.8 \%$ in the ticagrelor group. A major limitation of the trial involved the pathophysiological differences between the effect of ticagrelor and clopidogrel on inflammation and endothelial dysfunction in STEMI patients.

\section{PLATO (2009)}

The Platelet Inhibition and Patient Outcomes (PLATO) was an international, randomized trial that compared the efficacy of platelet inhibition of ticagrelor against prasugrel in patients with STEMI who underwent percutaneous coronary intervention. ${ }^{5}$ The primary endpoint was residual platelet reactivity 2 hours after a loading dose, while the secondary endpoints were percentage of high residual platelet reactivity, acute stent thrombosis and in-hospital bleeding after thrombolysis for MI. The study showed prasugrel was non-inferior to ticagrelor for residual platelet inhibition at 2 hours after the loading dose. PLATO trial entailed a similar limitation involving the differential effect of ticagrelor and prasugrel among STEMI patients.

\section{PEGASUS-TIMI 54 (2016)}

The Prior Heart Attack Using ticagrelor Compared to Placebo on a Background of Aspirin-Thrombolysis in Myocardial Infarction 54 (PEGASUS-TIMI 54), was an international, randomized, double-blinded, placebocontrolled trial which evaluated the efficacy of ticagrelor against a placebo among patients with ACS who underwent coronary artery bypass grafting $(\mathrm{CABG}){ }^{7}$ The primary outcome was graft occlusion at 3 months post $\mathrm{CABG}$ with secondary endpoints of MI, stroke and bleeding. Although the study was prematurely terminated, it did show a synergistic effect of ticagrelor with low-dose aspirin that reduced the risk of major adverse cardiovascular events. Furthermore, ticagrelor significantly reduced the risk of stroke, thus paving the way for its study and use among acute ischemic stroke (AIS) patients. A major limitation of the trial involved a lack of follow-up data that required extrapolation of clinical data at the time of the MI to estimate the trial eligibility.

\section{EUCLID (2017)}

The Examining Use of ticagrelor in Peripheral Artery Disease (EUCLID) trial was a double blinded, randomized prospective trial which evaluated the efficacy of clopidogrel against ticagrelor in patients with symptomatic peripheral vascular disease with a median follow-up of 30 months. ${ }^{6}$ The primary efficacy endpoint was cardiovascular or neurologic ischemic event and safety end point of major bleeding. The study concluded that ticagrelor was not superior to clopidogrel for reduction of cardiovascular events while the rates of major bleeding was similar in both the groups. One of the limitations involved in the EUCLID trial was a lack of inclusion of aspirin due to the constraints involved in the feasibility of conducting a three-group study and complications in blinding when dual antiplatelet therapy would be clinically warranted after randomization. Therefore, direct conclusions about the effect of the studied agents as compared with aspirin among patients with peripheral artery disease could not be evaluated.

\section{TREAT (2019)}

Ticagrelor in Patients With ST-Elevation Myocardial Infarction Treated With Pharmacological Thrombolysis (TREAT) was an international, multicenter, randomized, open-label with blinded endpoint adjudication trial to evaluate the efficacy of ticagrelor when compared with clopidogrel in STEMI patients treated with fibrinolytic therapy. The key outcomes were cardiovascular mortality, myocardial infarction, or stroke, and the same composite outcome with the addition of severe recurrent ischemia, transient ischemic attack, or other arterial thrombotic events at 12 months. The combined outcome was seen in $6.7 \%$ of the patients treated with ticagrelor and $7.3 \%$ in clopidogrel with $P=0.53$. The authors concluded that among patients age $<75$ years with STEMI, administration of ticagrelor after fibrinolytic therapy did not significantly reduce the frequency of cardiovascular events when compared with clopidogrel. The trial likely entailed a selection bias due to the lack of double blinding and did not carry adequate statistical power to accurately assess the safety and efficacy.

\section{Ticagrelor Use in Stroke Trials}

Various trials have investigated the efficacy of ticagrelor among patients with stroke or high-risk transient ischemic attack (TIA).

\section{SOCRATES (2016)}

The Acute Stroke or Transient Ischaemic Attack Treated with Aspirin or Ticagrelor and Patient Outcomes (SOCRATES) trial, was an international, randomized, double-blinded prospective trial that evaluated the efficacy of ticagrelor against aspirin among patients with TIA or AIS with an average of 3 months follow up. ${ }^{8}$ The primary outcome was the time to occurrence of stroke, myocardial infarction, or death within 90 days, and occurred in $6.7 \%$ of patients treated with 
ticagrelor versus $7.5 \%$ treated with aspirin $(P=0.07)$. Recurrent ischemic strokes occurred in 5.8\% in the ticagrelor group versus $6.7 \%$ in the aspirin group $(P=0.046)$. There was no statistical difference in major bleeding, intracranial hemorrhage, or fatal bleeding in either group.

A subgroup analysis of SOCRATES was performed that subsequently showed aspirin use in the pre-enrollment period, may actually have been substantial enough to confer a passive or residual protective effect and may have provided an additional benefit to the ticagrelor group, by conferring partial dual antiplatelet therapy. Among patients with large artery disease, ticagrelor was observed to be more efficacious than aspirin in reducing the rates of an ischemic event within 90 days among the patients with ipsilateral atherostenotic lesion. ${ }^{9}$ A few of the limitations in SOCRATES were: patients less than 40 years of age were excluded, lack of clinical follow up beyond 90 days, and a high rate of premature discontinuation of the use of ticagrelor due to either dyspnea or bleeding events.

\section{PRINCE (2018)}

The Platelet Reactivity in Acute Nondisabling Cerebrovascular Events (PRINCE) trial was a randomized multicenter prospective trial performed in China that primarily evaluated the platelet reactivity in the treatment groups involving ticagrelor plus aspirin and clopidogrel plus aspirin among the patients with TIA or minor AIS with an average 3 month follow up. ${ }^{10}$ The patients enrolled were categorized into carriers versus non-carriers of the CYP2C19 LOF allele genotype. Interestingly, in patients with large artery atherosclerosis, patients in the ticagrelor/aspirin group had a lower stroke recurrence rate at 90 days compared with the clopidogrel/aspirin group $(P=0.04)$. The authors reported that ticagrelor was superior to clopidogrel in inhibiting platelet reactivity, whereas no statistically significant interactions were noted between the carriers and non-carriers of the allele on platelet reactivity. One of the major limitations of the PRINCE trial was that the study population was enrolled from rural China, which limits the generalizability of results. Additionally, the trial was terminated early with only $50 \%$ of the projected sample recruited due to interim analysis by DSMB, thereby undercutting the power of the trial to study the clinical events.

\section{THALES (2020)}

More recently the Acute STroke or Transient IscHaemic Attack Treated With TicAgreLor and ASA for PrEvention of Stroke and Death (THALES) trial, was performed. ${ }^{11}$ It was a randomized, double-blinded, placebo-controlled prospective trial that compared the efficacy and safety of ticagrelor with aspirin among patients with mild-tomoderate acute non-cardioembolic ischemic strokes, with a National Institutes of Health Stroke Scale (NIHSS) score of 5 or less, or TIA and who were not undergoing thrombolysis or thrombectomy.

The primary outcome of composite of stroke or death within 30 days occurred in $5.5 \%$ of patients in the ticagrelor-aspirin group and in $6.6 \%$ in the aspirin monotherapy group $(P=0.02)$. Ischemic stroke occurred in $5.0 \%$ in the ticagrelor-aspirin group and in $6.3 \%$ in the aspirin group $(P=0.004)$. The incidence of disability did not differ significantly between the two groups as severe bleeding occurred in $0.5 \%$ of patients in the ticagreloraspirin group and only $0.1 \%$ of patients in the aspirin group $(P=0.001)$. A sub-group analysis of THALES trial involving stroke patients with ipsilateral stenosis investigated the primary endpoint i.e., time to the occurrence of stroke or death within 30 days. ${ }^{17}$ Patients randomized to ticagrelor achieved a lower rate $(8.1 \% \mathrm{v} / \mathrm{s} 10.9 \%)$ of primary endpoint. A few limitations of THALES were: absence of long-term data on the efficacy and safety beyond 30 days and study patient population was limited to Caucasians and Asians, limiting the generalizability of the results.

\section{Meta-Analyses}

A systemic review and meta-analysis involving 13 randomized controlled clinical trials $(\mathrm{RCT})(\mathrm{n}=64,360$ patients) was performed to compare the efficacy and safety of ticagrelor among patients with cerebral or cardiovascular risk factors. ${ }^{1}$ The authors observed that ticagrelor reduced the risk of ischemic stroke $(P=0.003)$, combined ischemic and hemorrhagic strokes $(P=0.05)$ and composite of stroke, MI, and cardiovascular death $(P=0.03)$. Ticagrelor was not associated with an increase in risk of mortality $(P=0.40)$ or major bleeding events $(P=0.19)$ among patients with prior history of ischemic stroke or TIA. Ticagrelor was observed to have a positive effect on primary and secondary stroke prevention, however, at the expense of side-effects including dyspnea, hyperuricemia, and major or minor bleeding events. However, the study did not observe any significant increase in major bleeding events with ticagrelor, suggesting the slightly increased risk of combined major or minor bleeding events was likely observed in patients with higher bleeding risk. ${ }^{1}$

Similarly, a meta-analysis involving 10 RCTs $(\mathrm{n}=$ 73,121 patients) compared the safety and efficacy of 
ticagrelor with traditional antiplatelet agents among patients with coronary or non-coronary atherothrombotic disease. ${ }^{12}$ In comparison to traditional antiplatelet therapies such as aspirin or clopidogrel, ticagrelor was associated with a significant reduction in mortality and recurrent cardiovascular events. These benefits were evident in studies involving patients with CAD while not in studies involving non-coronary atherothrombotic disease. The risk of cerebrovascular events was significantly reduced in the ticagrelor group, mainly driven by a significant benefit in stroke in patients with a previous non-coronary atherothrombotic event. Despite significant mortality benefit and decrease in recurrent ischemic events, ticagrelor was associated with an increase in major bleeding complications.

\section{Use of Ticagrelor as Dual Anti-Platelet Therapy}

Ticagrelor has been studied as both single and dual antiplatelet therapeutic medication to assess the safety and efficacy clinical outcomes. A systematic review and metaanalysis by Malhotra et al. ${ }^{1}$ performed a subgroup analysis to further evaluate the use of ticagrelor as dual antiplatelet therapy. The authors compared all the RCTs involving concomitant administration of antiplatelet agents and ticagrelor with ticagrelor monotherapy. In comparison to conventional dual antiplatelet therapy, there was a significant reduction of composite stroke, MI and cardiovascular death among patients treated with concomitant ticagrelor and conventional antiplatelet agent.

\section{Bleeding Complications}

In comparison to traditional antiplatelet agents, ticagrelor is associated with a higher rate of major and/or minor bleeding complications. Ticagrelor is associated with a higher rate of major bleeding, including fatal and intracranial hemorrhages, in comparison to clopidogrel monotherapy. ${ }^{4}$ The recently published THALES trial further demonstrated an increased risk of moderate to severe bleeding risk among patients treated with ticagrelor and aspirin as compared with aspirin alone $(0.5 \%$ vs $0.1 \%$ ), with a significantly higher rate of intracranial hemorrhage. ${ }^{11}$ Previous studies ${ }^{13}$ involving patients with acute coronary syndrome suggested a threefold increased risk of bleeding complications among patients treated with ticagrelor and aspirin in comparison to traditional antiplatelet regimens. The bleeding complications encompassed both major and major or minor bleeding events.

\section{Comparison of Ticagrelor with Other Antithrombotics}

Ticagrelor is not a prodrug that allows for rapid, potent, and consistent inhibition of platelet aggregation. These attractive pharmacologic, pharmacokinetic, and pharmacodynamic properties may have contributed to a significant reduction in thrombotic events in the PLATO trial. The ability of ticagrelor to alter adenosine uptake by red blood cells likely affects the efficacy and safety of the agent (Figure 1). Although direct comparison with prasugrel is difficult due to lack of comparative head-to-head trials, certain analyses could be made between these medications. Unlike prasugrel, ticagrelor offers advantage of usage regardless of the ACS management strategy (medical or invasive). There are no limitations for the use of ticagrelor based on clinical factors such as body weight, age or prior ischemic stroke. However, prasugrel has been observed to be more beneficial among patients with diabetes mellitus. Lastly, single day dosing of prasugrel is certainly an advantage in comparison to twice-daily dosing for ticagrelor.

\section{Practical Use, Costs and Benefits}

Based on the cost analysis data from the PLATO trial, concomitant use of ticagrelor and aspirin tends to increase

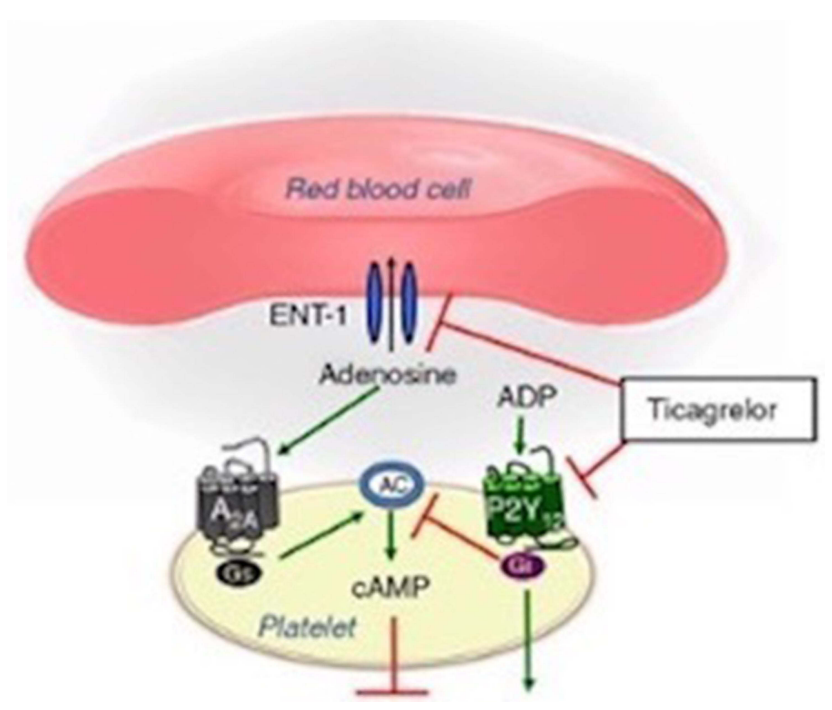

Figure I Mechanism of action of ticagrelor on platelet activation and aggregation. Notes: Adapted from Nylander S, Femia EA, Scavone M, et al. Ticagrelor inhibits human platelet aggregation via adenosine in addition to $\mathrm{P} 2 \mathrm{Y} 12$ antagonism. Journal of Thrombosis and Haemostasis. 2013;1 (10):1867-1876. (C) 2013 International Society on Thrombosis and Haemostasis. ${ }^{3}$ 
the life expectancy at an incremental cost that was well within the accepted benchmark of good value for money. Interestingly, in a 2015 drug cost analysis based on the National Average Drug Acquisition Cost, ${ }^{14}$ the average cost of ticagrelor was $\$ 7.88 /$ day compared with $\$ 0.11$ / day for clopidogrel. Although overall superiority was not established in either the THALES or SOCRATES trial, a subgroup analysis highlighted the efficacy of ticagrelor in a specific patient population which could potentially lead to lower average daily medication cost. As we understand how to better stratify and tailor our management based on patient demographics, stroke mechanism and various risk factors, ticagrelor has a potential to emerge as a more affordable antithrombotic choice for acute and chronic stroke prevention, and further expand the armamentarium of stroke neurologists.

\section{Current Use of Ticagrelor in Stroke Patients}

According to 2019 American Heart Association and American Stroke Association guidelines, ${ }^{15}$ ticagrelor is not recommended over aspirin for treatment of patients with minor stroke (NIHSS $\leq 5$ ) or high-risk TIA, however, the Food and Drug Administration has approved its use in patients with contraindications to aspirin. Additionally, the guidelines suggest that ticagrelor seems to be a reasonable alternative in stroke patients who have a contraindication to aspirin (Class III evidence). ${ }^{15}$ Although use of ticagrelor remains limited in the stroke field, it has been used among patients undergoing neuroendovascular procedures. An Indian study selected 32 patients from their cohort undergoing endovascular intervention that were non-responders to clopidogrel. ${ }^{16}$ All patients were treated with a $180-\mathrm{mg}$ loading dose of ticagrelor, followed by a scheduled dose of $90 \mathrm{mg}$ twice daily. Twenty patients $(63 \%)$ were treated for intracranial aneurysm, two $(6 \%)$ for dissecting aneurysms, nine $(28 \%)$ for stenotic lesions, and one $(3 \%)$ for carotidcavernous fistula. No patient experienced any adverse effects related to the use of ticagrelor in the postoperative period. The authors suggested ticagrelor as a safe alternative to clopidogrel non-responders. Although ticagrelor was reported to be safe and effective, a few disadvantages observed with the use of ticagrelor were (a) higher cost in comparison to traditional antiplatelet agents, (b) twice-daily dosing, and (c) potential risk of rapid platelet aggregation after discontinuation.

\section{Future Direction}

The use of ticagrelor is expected to increase as additional trials continue to investigate its use in varied patient populations, and assess the efficacy and safety demonstrated in earlier trials. A study from Kafrelsheikh University is investigating treatment with ticagrelor within 9 hours of acute ischemia. ${ }^{18}$ Similarly, CHANCE 2 trial is currently recruiting patients to assess the effects of ticagrelor plus aspirin versus clopidogrel plus aspirin on reducing the 3 -month risk of any stroke (both ischemic and hemorrhagic) as a primary outcome when initiated within 24 hours of symptom onset among patients with TIA or minor stroke and who are CYP2Y19 LOF alleles carriers. ${ }^{19}$ Future RCTs are warranted to evaluate the safety profile of ticagrelor among ischemic stroke patients who are eligible for acute thrombolytic and endovascular therapies.

\section{Conclusion}

Primary and secondary prevention with antiplatelet therapy continues to be an integral part of stroke management. Randomized controlled clinical trials thus far have highlighted the benefits of ticagrelor in certain sub-population $\mathrm{s}$ with vascular risk factors. Further studies designed to assess the superiority of ticagrelor compared with other antiplatelets could broaden its use in acute stroke care. The limitation thus far is majorly related to bleeding complications and drug cost, however, with better understanding of its mechanism, future studies could provide additional data that could advance the use of ticagrelor in both acute and chronic stroke care.

\section{Author Contributions}

All authors made a significant contribution to the work reported, whether that is in the conception, study design, execution, acquisition of data, analysis and interpretation, or in all these areas; took part in drafting, revising or critically reviewing the article; gave final approval of the version to be published; have agreed on the journal to which the article has been submitted; and agree to be accountable for all aspects of the work.

\section{Funding}

This study received no specific grant from any funding agency in the public, commercial or not-for-profit sectors.

\section{Disclosure}

The authors report no conflicts of interest in this work. 


\section{References}

1. Malhotra K, Goyal N, Kasunich AS, et al. Ticagrelor for stroke prevention in patients with vascular risk factors: a systematic review and meta-analysis. J Neurol Sci. 2018;390:212-218. doi:10.1016/j. jns.2018.05.001

2. Dobesh PP, Oestreich JH. Ticagrelor: pharmacokinetics, pharmacodynamics, clinical efficacy, and safety. Pharmacother J Hum Pharmacol Drug Ther. 2014;34(10):1077-1090. doi:10.1002/phar.1477

3. Nylander S, Femia EA, Scavone M, et al. Ticagrelor inhibits human platelet aggregation via adenosine in addition to P2Y12 antagonism. J Thromb Haemost. 2013;11(10):1867-1876. doi:10.1111/jth.12360

4. Wallentin L, Becker RC, Budaj A, et al. Ticagrelor versus clopidogrel in patients with acute coronary syndromes. $N$ Engl J Med. 2009;361 (11):1045-1057. doi:10.1056/NEJMoa0904327

5. Mahaffey KW, Wojdyla DM, Carroll K, et al. Ticagrelor compared with clopidogrel by geographic region in the platelet inhibition and patient outcomes (PLATO) trial. Circulation. 2011;124(5):544-554. doi:10.1161/CIRCULATIONAHA.111.04749

6. Hiatt WR, Fowkes FG, Heizer G, et al. Ticagrelor versus clopidogrel in symptomatic peripheral artery disease. $N$ Engl J Med. 2017;376 (1):32-40. doi:10.1056/NEJMoa1611688

7. Bonaca MP, Goto S, Bhatt DL, et al. Prevention of stroke with Ticagrelor in patients with prior myocardial infarction: insights from PEGASUS-TIMI 54 (Prevention of Cardiovascular Events in Patients With Prior Heart Attack Using Ticagrelor Compared to Placebo on a Background of Aspirin-Thrombolysis in Myocardial Infarction 54). Circulation. 2016;134(12):861-871. doi:10.1161/ CIRCULATIONAHA.116.024637

8. Johnston SC, Amarenco P, Albers GW, et al. Ticagrelor versus aspirin in acute stroke or transient ischemic attack. $N$ Engl J Med. 2016;375 (1):35-43. doi:10.1056/NEJMoa1603060

9. Wong KSL, Amarenco P, Albers GW, et al. Efficacy and safety of Ticagrelor in relation to aspirin use within the week before Randomization in the SOCRATES Trial. Stroke. 2018;49 (7):1678-1685. doi:10.1161/STROKEAHA.118.02055

10. Wang Y, Chen W, Lin Y, et al. Ticagrelor plus aspirin versus clopidogrel plus aspirin for platelet reactivity in patients with minor stroke or transient ischaemic attack: open label, blinded endpoint, randomised controlled Phase II trial. BMJ. 2019;365:12211. doi:10.1136/ bmj.12211
11. Johnston SC, Amarenco P, Denison H, et al. Ticagrelor and aspirin or aspirin alone in acute ischemic stroke or TIA. $N$ Engl J Med. 2020;383(3):207-217. doi:10.1056/NEJMoa1916870

12. Verdoia M, Kedhi E, Suryapranata H, De Luca G. Ticagrelor in the prevention of coronary and non-coronary atherothrombotic events: a comprehensive meta-analysis of 10 randomized trials. Atherosclerosis. 2019;284:136-147. doi:10.1016/j.atherosclerosis.2019.02.011

13. Charpentier T, Ferdynus C, Lair T, et al. Bleeding risk of ticagrelor compared to clopidogrel in intensive care unit patients with acute coronary syndrome: a propensity-score matching analysis. PLoS One. 2020;15(5):e0232768. doi:10.1371/journal.pone.0232768

14. Cowper PA, Pan W, Anstrom KJ, et al. Economic analysis of Ticagrelor therapy from a U.S. perspective. $J$ Am Coll Cardiol. 2015;65(5):465-476. doi:10.1016/j.jacc.2014.11.034

15. Powers WJ, Rabinstein AA, Ackerson T, et al. Guidelines for the Early Management of Patients With Acute Ischemic Stroke: 2019 Update to the 2018 Guidelines for the Early Management of Acute Ischemic Stroke: a Guideline for Healthcare Professionals From the American Heart Association/American Stroke Association. Stroke. 2019;50(12). doi:10.1161/STR.0000000000000211

16. Karan V, Vyas D, Bohra V, Huded V. Ticagrelor use in Indian patients undergoing neuroendovascular procedures: a single center experience. Neurointervention. 2019;14(2):125-130. doi:10.5469/ neuroint.2019.00087

17. Amarenco P, Denison H, Evans SR, et al.; THALES Steering Committee and Investigators*. Ticagrelor added to aspirin in acute nonsevere ischemic stroke or transient ischemic attack of atherosclerotic origin. Stroke. 2020;51(12):3504-3513. PMID: 33198608; PMCID: PMC7678660. doi:10.1161/STROKEAHA.120.032239

18. Ticagrelol versus aspirin inischemic stroke. ICHGCP; n.d. Available from: https://ichgcp.net/clinical-trials-registry /NCT03884530. Accessed June 1, 2021.

19. Clopidogrel with aspirin in high-risk patients with acute non-disabling cerebrovascular events II; n.d. Available from: https:// clinicaltrials.gov/ct2/show/NCT04078737. Accessed June 1, 2021.
Vascular Health and Risk Management

\section{Publish your work in this journal}

Vascular Health and Risk Management is an international, peerreviewed journal of therapeutics and risk management, focusing on concise rapid reporting of clinical studies on the processes involved in the maintenance of vascular health; the monitoring, prevention and treatment of vascular disease and its sequelae; and the involvement of metabolic disorders, particularly diabetes. This journal is indexed on PubMed Central and MedLine. The manuscript management system is completely online and includes a very quick and fair peerreview system, which is all easy to use. Visit http://www.dovepress. com/testimonials.php to read real quotes from published authors. 